\title{
Grain rotations in ultrafine-grained aluminum films studied using in situ TEM straining with automated crystal orientation mapping
}

\author{
Ehsan Izadi ${ }^{1}$, Amith Darbal ${ }^{2}$, Rohit Sarkar ${ }^{3}$ and Jagannathan Rajagopalan ${ }^{1,3^{*}}$ \\ 1. Department of Mechanical and Aerospace Engineering, School for Engineering of Matter Transport and \\ Energy, Arizona State University, Tempe 85281, USA. \\ 2. Nanomegas USA, Tempe, AZ, USA. \\ 3. Department of Materials Science and Engineering, School for Engineering of Matter Transport and Energy, \\ Arizona State University, Tempe 85281, USA.
}

\begin{abstract}
In situ TEM straining allows probing deformation mechanisms of ultrafine-grained and nanocrystalline metals. While obtaining statistically meaningful information about microstructural changes using conventional bright-field/dark-field imaging or diffraction is time consuming, automated crystal orientation mapping in TEM (ACOM-TEM) enables tracking orientation changes of hundreds of grains simultaneously. We use this technique to uncover extensive grain rotations during in situ tensile deformation of a freestanding, ultrafine-grained aluminum film (thickness $200 \mathrm{~nm}$, mean grain size $180 \mathrm{~nm}$ ). During loading, both the fraction of grains that undergo rotations and the magnitude of their rotations increase with strain. The rotations are partially or fully reversible in a significant fraction of grains during unloading, leading to notable inelastic strain recovery. More surprisingly, the direction of rotation remains unchanged for a small fraction of grains during unloading, despite a sharp reduction in the applied stress. The ACOM-TEM measurements also provide evidence of reversible and irreversible grain/twin boundary migrations in the film. These microstructural observations point to a highly inhomogeneous and constantly evolving stress distribution in the film during both loading and unloading.
\end{abstract}

Keywords: In situ TEM; Automated crystal orientation map; Reversible grain rotation; Bauschinger effect; Grain boundary migration; Detwinning

*Corresponding author: Email: jrajago1 @asu.edu (J. Rajagopalan). 


\section{Introduction}

It is well known that grain refinement is a powerful tool to enhance the performance of metallic materials. In terms of mechanical properties, nanocrystalline (NC) and ultrafine-grained (UFG) metal films are known to exhibit higher yield and fatigue strength, improved corrosion resistance and enhanced superplasticity [1]-[6] compared to their coarse-grained counterparts. There is also extensive experimental evidence which shows that the mechanical behavior of UFG/NC metals is highly rate sensitive [7], [8] and that the strain rate sensitivity (SRS) considerably increases as the grain size becomes finer [9]-[12]. In addition to grain size, texture has also been shown to significantly influence the mechanical behavior of UFG/NC metal films [13]-[16]. Experimental investigations on UFG Al films with similar thickness and mean grain sizes but different textures have revealed notable changes in flow stress and inelastic strain recovery during unloading [13], [15]. Similarly, it has been reported that the variation of texture in NC Ni foils leads to a distinct change of the yield stress and ultimate strength [8].

Since the changes in the macroscopic behavior of NC/UFG metals result from the changes in the underlying mechanisms, various in situ experimental techniques have been employed to obtain a detailed understanding of their deformation mechanisms. Among these techniques, in situ synchrotron X-ray diffraction has been used to track the evolution of micro strain and changes in grain size and texture during deformation [16], [17]. However, it is not possible to directly resolve the microstructure of UFG/NC metals with this technique [18]. In situ bright-field/darkfield transmission electron microscopy (TEM) has provided considerable insight into generation and motion of dislocations and deformation induced grain growth [14], [19], but these techniques do not yield meaningful statistics about microstructural evolution. In situ scanning electron 
microscope (SEM) experiments with automated analysis of electron backscattered diffraction (EBSD) can also be used to analyze the texture and crystallography of grain boundaries [20][22]. The limited spatial resolution in orientation imaging using SEM-EBSD, however, makes it difficult to investigate microstructural changes in UFG/NC materials [23], [24].

Alternatively, automated crystal orientation mapping in TEM (ACOM-TEM) using precession electron diffraction, a recently developed technique, can be used to perform crystallographic analyses on UFG/NC metals during in situ straining [18], [22], [25], [26]. In the ACOM-TEM technique, which provides a spatial resolution better than $3 \mathrm{~nm}$, a precessing nanoprobe electron beam is scanned over the specimen to collect spot diffraction patterns with reduced dynamical effects and improved pattern quality. The diffraction patterns are then automatically indexed using a template matching process, following which the orientation maps of the sample are extracted [24]. ACOM-TEM enables direct acquisition of orientation/phase map over micrometer sized areas while enhancing the ability to identify grains, microtexture and twin and other coincidence site lattice boundaries [24]. This technique is especially helpful in obtaining a comprehensive picture of microstructural evolution in UFG/NC metals, which are known to have an unstable microstructure [18]. Precession electron diffraction has also been used to estimate dislocation densities and driving force for twin formation using Nye tensor [27], [28].

The primary objective of this study was to understand the deformation mechanisms in UFG Al films that have a random orientation of grains (no preferred texture). The study was motivated by recent experiments which showed that non-textured UFG Al films have an unusually large SRS exponent $(\mathrm{m}=0.107)$, possibly caused by time dependent grain rotations [15]. Here, we used a 
combination of in situ and ex situ quasi-static, load-unload experiments to further explore the deformation behavior of such films. Specifically, we used in situ tensile straining with ACOMTEM to monitor the changes in grain orientations during deformation and obtain quantitative information about the magnitude and nature of such rotations.

The ACOM-TEM analysis provides evidence of extensive grain orientation changes in the UFG Al film during loading, with both the fraction of grains that undergo rotations and the magnitude of their rotations increasing with strain. The rotations are partially or fully reversible in a significant fraction of the grains during unloading, leading to notable inelastic strain recovery (Bauschinger effect). More surprisingly, a small fraction of grains continue to rotate in the same direction during unloading, even when the applied stress has been reduced significantly. The ACOM results also reveal several unusual phenomena including reversible grain boundary migration and detwinning during loading and unloading.

\section{Experimental details}

\subsection{Thin film synthesis and characterization}

A $200 \mathrm{~nm}$ thick Al film was deposited on 200- $\mu \mathrm{m}$ thick, $100 \mathrm{~mm}$ diameter, (001)-oriented silicon (Si) wafers using DC magnetron sputtering. The native silicon dioxide layer on the Si wafer was left intact, which prevented epitaxial growth of $\mathrm{Al}$ and resulted in a film with random orientation of grains. A statistical analysis of bright field images of the Al film, obtained using a JEOL 2010F TEM, revealed a mean grain size of $180 \mathrm{~nm}$ (Fig. 1). An analysis of the selected area diffraction pattern showed no clear evidence of a preferred texture. Based on the ACOM map, 
twin boundaries (primarily $\Sigma 3$ ) comprised about $10 \%$ of the total grain boundary length in the asdeposited film. A similar fraction of twin boundaries has been previously reported on vapor deposited UFG Al films [29].

\subsection{Sample fabrication}

Dog-bone shaped freestanding samples of the film were co-fabricated with a micro-electromechanical-system (MEMS) based tensile testing device (Fig. 2a) using photolithography and reactive ion etching techniques described in [30]. The MEMS devices include three built-in gauges, G1, G2 and G3, to track the force and deformation on the freestanding sample. A custom MATLAB $^{\mathrm{TM}}$ program was used to track prescribed features across a series of images of the gauges using cross-correlation techniques to measure the displacement of the gauges. The displacement of G1 with respect to $\mathrm{G} 2\left(\Delta \mathrm{X}_{d}\right)$, which corresponds to the deformation of the sample, was used to obtain the sample strain whereas the displacement of G2 with respect to G3 $\left(\Delta \mathrm{X}_{F}\right)$, which gives the deflection of the force-sensing beams, was used to calculate the stress on the sample.

As evident from Fig. 2a, the sample is arranged in series with the force-sensing beams and hence the force on sample and the force-sensing beams is the same. Therefore, multiplying $\Delta \mathrm{X}_{F}$ by the stiffness of the forces-sensing beams, which was measured in a separate experiment, gives the net force and thus the stress on the sample. The error in strain measurement using the MATLAB${ }^{\mathrm{TM}}$ based image-processing technique was less than $0.003 \%$ whereas the uncertainty in stress measurement, which came from the error in calibrating the force sensing beams and the variation in sample thickness was found to be about $10 \mathrm{MPa}$. The $\mathrm{Al}$ film was under compressive stress in 
the as-deposited state and, therefore, the freestanding samples buckled when they were released from the Si substrate. However, the samples were almost macroscopically stress-free before loading since the stress required for buckling along the length is very low $(<0.1 \mathrm{MPa})[13]$.

\subsection{Tensile testing}

The mechanical behavior of the thin film specimens was investigated through ex situ and in situ TEM tensile load-unload experiments. The averaged strain rate in both in situ and ex situ experiments was less than $10^{-5} / \mathrm{s}$, resulting in quasi-static loading and unloading. To eliminate possible variations in mechanical behavior that arise from sample size effects, the width $(75 \mu \mathrm{m})$ and length $(375 \mu \mathrm{m})$ of all samples were kept constant.

For the in situ TEM tensile experiment, the MEMS device was mounted on a displacement controlled single tilt straining holder (Fig. 2b) and loaded on to a JOEL ARM200F TEM equipped with the Nanomegas ASTAR system for ACOM data acquisition. Strain pulses (typically corresponding to $<0.1 \%$ strain) were applied to the sample and the stress-strain data was recorded after allowing the film to relax for two minutes. The displacement applied on the MEMS device was kept constant when ACOM-TEM data was acquired. The data was collected from the same $3 \mu \mathrm{m} \times 3 \mu \mathrm{m}$ area at three strain levels during loading and two strain levels during unloading with a step size of $10 \mathrm{~nm}$. It is relevant to note that the number of grains (325) in the scanning area is sufficiently large to provide meaningful statistics but is still small compared to the total number of grains in the sample $(\sim 800,000)$. Thus, electron beam-induced relaxation [31] in the scanning area is unlikely to change the overall stress-strain response. 
Before scanning, an electron probe with $\sim 1 \mathrm{~nm}$ diameter was generated using spot size 4 and 10 $\mu \mathrm{m} \mathrm{C} 2$ aperture,. The spot diffraction patterns were then obtained using a beam precession angle of $0.4^{\circ}$, and a camera length of $120 \mathrm{~mm}$. Finally, the indexing of the acquired ACOM-TEM data was performed by matching the spot diffraction patterns with a bank of templates for aluminum using the ASTAR software package to generate the crystal orientation maps. Note that the template matching process considers both the location and relative intensities of the diffraction spots and results in an angular resolution of $\sim 0.3^{\circ}[32]$.

The ACOM maps provided information of the Euler angles, cross correlation index and reliability index for the scanned points. After all the orientation maps were aligned with respect to each other, the analyses were done only on grains with a reliability index greater than 15 (Supplementary Fig. 1), as suggested in [32]. Based on this criterion, 31 of the 325 grains in the scanning area were found to be unreliably indexed and their data was discarded. In addition, 44 grains had a large spread in the point-to-point orientation (standard deviation $>0.1^{\circ}$ ) and were also not considered for analysis. For the remaining 250 grains, mean grain orientations were calculated by averaging the orientations of the all the points within each grain. These mean grain orientations were used for all further analysis.

\section{Results}

\subsection{Ex situ experiments}

The stress-strain response from the ex-situ load-unload experiment on the $200 \mathrm{~nm}$ thick nontextured $\mathrm{Al}$ film is plotted in Fig. 3a. The film showed significant deviation from elastic behavior 
towards the end of unloading, leading to early Bauschinger effect (BE). To quantify this Bauschinger strain, we calculated the $\mathrm{BE}$ ratio $\left(\varepsilon_{\mathrm{B}} / \varepsilon_{\mathrm{p}}\right)$ adopting the notations described in Fig. 3a. Briefly, $\varepsilon$ represents the total strain during loading, $\varepsilon_{\mathrm{p}}$ the expected plastic strain if the sample showed elastic unloading, and $\varepsilon_{\mathrm{B}}$ the recovered strain (the difference between the expected and actual plastic strain). The BE ratio for the non-textured film was 0.27 . For completeness, we also show the stress-strain response of another non-textured film at different strain rates [15] in Fig. 3b. As evident from the data, the flow stress (defined as stress at $0.9 \%$ strain) of the non-textured film increased by more than $90 \%$ (from $243 \mathrm{MPa}$ to $473 \mathrm{MPa}$ ) as the strain rate was increased from $6.8 \times 10^{-6} / \mathrm{s}$ to $6.7 \times 10^{-3} / \mathrm{s}$.

\subsection{In situ ACOM-TEM experiments}

Figure 4a shows the stress-strain response of the $200 \mathrm{~nm}$ non-textured film during in situ TEM straining. As indicated on the stress-strain curve, the orientation maps were obtained at $0.7 \%$, $1.3 \%$ and $1.9 \%$ strain during loading (from here on referred to as $0.7 \% \mathrm{~L}, 1.3 \% \mathrm{~L}$ and $1.9 \% \mathrm{~L}$ ), and at $1.7 \%$ and $1.6 \%$ strain during unloading (from here on referred to as $1.7 \%$ UL and $1.6 \%$ UL). At all these scan points, it took between 15-30 minutes to switch the TEM from imaging to scanning mode and another 30 minutes to subsequently perform the scan. This led to significant time-dependent stress relaxation at each of the scanning points during loading. Nevertheless, we tried to minimize electron beam induced relaxation by using a $200 \mathrm{kV}$ beam with low intensity, as suggested in [31]. We also note that since the scan is performed point-by-point and the probe size is very small ( $1 \mathrm{~nm}$ diameter), the area illuminated at any instant is negligible compared to either the average grain size $(180 \mathrm{~nm})$ or the dimensions of the sample (75 $\mu \mathrm{m}$ wide, $375 \mu \mathrm{m}$ long). Therefore, we expect that beam induced artifacts are minimal. 
Using the mean orientation of each grain at different loading and unloading points, a systematic analysis was done to investigate their orientation changes. To quantify the grain rotations, we first calculated the rotation matrix required to transform the crystal coordinate axes ([100], [010] and [001] directions) of each grain from its current configuration to its reference configuration (at $0.7 \%$ strain). In other words, we compare the orientations of the same grain at two different points during deformation. Then, we converted this coordinate axes transformation for each grain into rotations about different axes. The nomenclature used for these rotations is described next.

For each grain $i, \theta_{i}$ is the total rotation about an arbitrary axis (Euler rotation theorem) required to transform the grain from its current configuration to its reference configuration, as schematically depicted in Fig. 5. We further decomposed $\theta$ into two rotations - first, about the out-of-plane normal to the film (parallel to the electron beam direction), followed by a rotation about an arbitrary direction in the plane of the film, as shown in Fig. 5. We designated the rotation of each grain about the film normal $\left(\psi_{i}\right)$ as in-plane rotation and the subsequent rotation $\left(\varphi_{i}\right)$ as the out-of-plane rotation. We also calculated the mean values of these quantities by considering only grains that had non-zero rotations. These mean values are indicated by $\theta_{\text {avg }}$, $\psi_{\text {avg }}$ and $\varphi_{\text {avg. }}$. The error in the rotation angles computed for individual grains is similar to the angular resolution of the system $\left(0.3^{\circ}\right)$. Hence, grains that showed rotations less than $0.3^{\circ}$ were considered to have zero rotation. The error for the mean quantities is significantly lower (better than $0.03^{\circ}$ ) because of averaging over hundreds of individual measurements.

To ensure that rigid body rotation of the film did not cause the grain orientation changes, the following steps were taken. The grain orientation maps were acquired from an area close to the midpoint (width-wise) of the film and away from the sample ends so that the deformation was 
uniaxial. Furthermore, the reference scan was taken after a sufficiently large strain $(0.7 \%)$ had been imposed to ensure that the initial buckling in the film was completely removed. For the same reason, the scans during unloading were also taken well before the film was fully unloaded. It should be noted that during both loading and unloading roughly $20-40 \%$ of the grains exhibited no change in their orientation, which confirms that there was no global rotation or tilt of the film.

Figure 6 provides a histogram of grain orientation changes that occurred during loading and unloading. As evident from the figure, the number of grains experiencing rotations increased from 144 to 193 as strain was increased from $1.3 \%$ to $1.9 \%$ during loading. In addition, the magnitude of rotation $(\theta)$ of the grains also increased. While the average $\theta$ (excluding grains that did not undergo any rotation) was about $1.4^{\circ}$ when the strain was increased from $0.7 \%$ to $1.3 \%$, it increased to $1.6^{\circ}$ when the strain was increased to $1.9 \%$.

When the sample was unloaded to $1.7 \%$ strain the $\theta_{\text {avg }}$ decreased to $1.5^{\circ}$. But surprisingly, it increased again to $1.6^{\circ}$ when the sample was further unloaded to $1.6 \%$ strain. Figure 7 shows the in-plane and out-of-plane rotations of the grains at $1.9 \%$ strain during loading. The average inplane rotation $\left(1.3^{\circ}\right)$ was slightly higher compared to the out-of-plane rotation $\left(1.1^{\circ}\right)$. However, the fraction of grains undergoing in-plane and out-of-plane rotations was roughly similar.

In addition to grain rotations, the in situ ACOM-TEM experiment also revealed some unusual microstructural changes. Several grains showed an increase/decrease in size during loading, which is consistent with previous studies on deformation induced grain growth in UFG and NC metals [18], [33]. However, unlike previous reports, we observed changes in grain size even during unloading, when the applied stress had been considerably reduced. Figure 8 and 9 provide 
two different examples of this phenomenon. Figure 8 shows a grain that reduced in size while loading, continued to shrink during unloading, and was completely annihilated. Figure 9, in contrast, shows a grain that exhibited reversible growth. The size of this grain increased during loading (from $\sim 9300 \mathrm{~nm}^{2}$ to $\sim 12400 \mathrm{~nm}^{2}$ ) but shrunk (to $6700 \mathrm{~nm}^{2}$ ) when the sample was unloaded. We also observed detwinning during both loading and unloading in some grains, as shown in Fig. 10.

\section{Discussion}

The experiments on non-textured UFG Al films reveal high strain rate sensitivity, and a large Bauschinger effect during quasi-static load-unload experiments. The ACOM-TEM measurements provide evidence of extensive grain rotations during loading. Unexpectedly, significant microstructural changes (grain rotation, grain annihilation, detwinning) also occur during unloading. These observations point to a highly heterogeneous deformation and a continuous redistribution of stresses in the film, as we discuss below.

Since the film has no preferred texture, loading occurs along a random crystallographic direction for each grain. Thus, depending on the orientation of the grain with respect to the loading axis, the elastic modulus can vary from $63.7 \mathrm{GPa}$ ([100] direction) to $76 \mathrm{GPa}$ ([111] direction). This would result in elastic strain mismatch between the grains even before the grains have begun to deform plastically. More importantly, when the grains start to deform plastically, the variation in the maximum Schmid factor of the grains, which ranges from 0.27 to 0.5 for uniaxial loading, leads to considerable difference in the resolved shear stress required to activate slip. In addition, 
the critical resolved shear stress required to activate slip is likely to be higher for relatively smaller grains compared to larger grains.

The combination of these factors (variation in grain size and Schmid factor) will result in considerable stress difference and plastic strain mismatch between plastically soft (large size and high Schmid factor) and hard grains (small size and low Schmid factor) as the strain is increased. Therefore, neighboring grains need to bend or rotate with respect to each other to maintain strain compatibility. The back stresses resulting from the inhomogeneous stress distribution would also lead to reverse yielding in the plastically soft grains during unloading, which would manifest as inelastic strain recovery (Bauschinger effect). Previous studies on UFG aluminum films have, indeed, revealed substantial back flow (reverse dislocation motion) during cyclic loadingunloading experiments [34]. The reverse motion of dislocations were shown to occur either due to back stresses arising from grain boundary pile-ups or because the dislocations were not fully inserted into the grain boundaries during loading.

The results from ACOM-TEM experiment are consistent with the above description of the deformation behavior. As shown in Fig. 6, both the number of grains that undergo orientation changes and the magnitudes of their rotation increase with strain during loading. Note that grain rotations could be accommodated by a combination of elastic and plastic deformation. The plastic deformation associated with grain rotation is likely to be mediated by dislocations in UFG metals, as shown in previous studies [35]. The presence of several grains with large misorientation gradients, which require geometrically necessary dislocations, in our sample also points to the same conclusion. 
Notably, during unloading the average grain rotation $\left(\theta_{\text {avg }}\right)$ first decreases to $1.5^{\circ}$ and then increases to $1.6^{\circ}$, which can be understood as follows. During the initial stages of unloading, the stresses in all the grains reduce and the rotations induced by elastic incompatibility between the grains are reversed. Upon further unloading, however, plastically soft grains undergo reverse yielding because of stress reversal, which manifests as the macroscopic Bauschinger effect. This reverse yielding leads to a reduction of back stresses in these soft grains and redistributes the stresses in their neighborhood, which necessitates further rotation of the surrounding grains to maintain compatibility. As a result, $\theta_{\text {avg }}$ again increases.

To verify if this is true, we analyzed the rotations of the grains during unloading with respect to the end of loading (1.9\% strain). But because the rotation axis is not constant at different loading and unloading points even for the same grain, it is not appropriate to directly measure the difference in $\theta$. However, such a comparison is more meaningful for in-plane rotations $(\psi)$ because they are always defined with respect to the film normal. Therefore, we decomposed the rotations into in-plane and out-of-plane components and analyzed the in-plane rotations as follows. If a grain rotated in the same direction about the film normal during both loading and unloading, we classify those rotations as forward rotations. If the sense of rotation during unloading was opposite to that of loading, we denote them as reverse rotations. Finally, if a grain showed no rotation during loading but had non-zero rotation during unloading, we classify those as uncorrelated rotations. The relative in-plane rotations $\left(\psi_{\text {rel }}\right)$ of the grains based on this classification scheme are plotted in Fig. 11. 
The results show that the proportion of grains that exhibit relative in-plane rotation (with respect to end of loading) increased as the sample was unloaded further. Specifically, the number of grains that underwent reverse rotations increased from 54 to 60 as the strain was decreased from $1.7 \%$ to $1.6 \%$. More importantly, the number of grains that undergo uncorrelated rotations increased even more (from 32 to 51). Such uncorrelated grain rotations, as argued earlier, are consistent with redistribution of stresses triggered by reverse yielding of plastically soft grains. Surprisingly, the number of grains that exhibit forward rotations also increased (from 11 to 20) with unloading. The forward rotations suggest that the local stress state remains similar for these grains during both loading and unloading, even though the macroscopic stress is substantially different.

The presence of grain rotations during unloading is also consistent with the qualitative observations of the evolving microstructure. As shown in Fig. 8, 9 and 10 there is evidence of both reversible and irreversible migration of grain/twin boundaries. It has been shown that these grain boundary migrations are primarily driven by the local stress state [36] and have been attributed to shear-coupled grain boundary motion [37]. Therefore, a reverse migration of a grain boundary during unloading (Fig. 9) likely reflects a reversal in the local stress state. In contrast, the continued migration of a grain/twin boundary (Fig. 8 and 10) would suggest that the local stress state remains similar to that during loading. While previous studies have reported grain rotation and growth in UFG and NC metals during loading [18], [35], our observations strongly imply that both grain orientations and the microstructure continue to evolve during unloading. This continued microstructural evolution could have significant implications for the fatigue behavior of UFG/NC metal films, which are used in MEMS resonators and switches [38], [39]. 
Recently developed methods [40] for in situ TEM fatigue testing provide a route to investigate these effects in greater detail.

Finally, we would like to note that the fraction of grains observed to undergo rotations in our UFG aluminum film is significantly larger compared to previous reports on UFG aluminum films [25], [35], even though the applied strain is substantially lower in our case (2\% compared to 6-7\% in [25] and [35]). This disparity is most likely caused by differences in the texture of the films that were investigated. The films in both [25] and [35] had a strong (111) texture, whereas our film had no preferred texture. A (111) texture is likely to make the deformation more homogeneous for two reasons. The (111) plane is transversely isotropic (same Young's modulus in all directions) and therefore elastic strain mismatch between grains is minimized. The (111) texture also leads to a significantly narrower range of maximum Schmid factors (0.408 to 0.471$)$ for the grains compared to a non-textured film (0.27 to 0.5$)$, which drastically reduces the heterogeneity in stress distribution induced by plastic anisotropy. As a result, there is much less need for grain rotation in a (111) textured film to maintain strain compatibility.

\section{Conclusions}

The deformation behavior of a non-textured UFG aluminum film was studied using quasi-static ex situ and in situ ACOM-TEM load-unload experiments. An analysis of the ACOM data revealed extensive grain orientation changes during deformation. During loading, the number of grains that undergo rotations increased with strain, and at $1.9 \%$ strain more than $75 \%$ of the grains had experienced rotations greater than $0.3^{\circ}$. These pervasive grain rotations, which are time-dependent [15], can explain the high strain rate sensitivity of non-textured Al films. 
During unloading, more than $52 \%$ of the grains experienced in-plane rotations as the sample strain was decreased from $1.9 \%$ to $1.6 \%$. Among these grains, about $46 \%$ underwent reverse rotations. The rest exhibited uncorrelated rotations (39\%) or forward rotations (15\%), which is consistent with redistribution of stresses triggered by reverse yielding of plastically soft grains. Overall, the microstructural observations point to a spatially inhomogeneous stress distribution in the film that constantly evolves during both loading and unloading. From a broader perspective, the results demonstrate how the combination of in situ straining and ACOM-TEM can provide a quantitative description of the microstructural evolution in UFG metals during deformation.

\section{Acknowledgements}

This material is based upon work supported by the National Science Foundation under awards CMMI 1400505, DMR 1454109 and CMMI 1563027. The authors would like to gratefully acknowledge the use of facilities at the John M. Cowley Centre for High Resolution Electron Microscopy and the Centre for Solid State Electronics Research at Arizona State University. 


\section{References}

[1] M. A. Meyers, A. Mishra, and D. J. Benson, "Mechanical properties of nanocrystalline materials," Prog. Mater. Sci., vol. 51, no. 4, pp. 427-556, May 2006.

[2] J. C. M. Li, Mechanical Properties of Nanocrystalline Materials. CRC Press, 2011.

[3] I. P. Semenova, A. V. Polyakov, G. I. Raab, T. C. Lowe, and R. Z. Valiev, "Enhanced fatigue properties of ultrafine-grained Ti rods processed by ECAP-Conform," J. Mater. Sci., vol. 47, no. 22, pp. 7777-7781, Jul. 2012.

[4] S. Lee, A. Utsunomiya, H. Akamatsu, K. Neishi, M. Furukawa, Z. Horita, and T. G. Langdon, "Influence of scandium and zirconium on grain stability and superplastic ductilities in ultrafine-grained Al-Mg alloys," Acta Mater., vol. 50, no. 3, pp. 553-564, Feb. 2002.

[5] E. A. Korznikova, S. Y. Mironov, A. V. Korznikov, A. P. Zhilyaev, and T. G. Langdon, "Microstructural evolution and electro-resistivity in HPT nickel," Mater. Sci. Eng. A, vol. 556, pp. 437-445, Oct. 2012.

[6] H. S. Kim, S. J. Yoo, J. W. Ahn, D. H. Kim, and W. J. Kim, "Ultrafine grained titanium sheets with high strength and high corrosion resistance," Mater. Sci. Eng. A, vol. 528, no. 29-30, pp. 8479-8485, Nov. 2011.

[7] L. Lu, S. X. Li, and K. Lu, "An abnormal strain rate effect on tensile behavior in nanocrystalline copper," Scr. Mater., vol. 45, no. 10, pp. 1163-1169, Nov. 2001.

[8] F. Dalla Torre, H. Van Swygenhoven, and M. Victoria, "Nanocrystalline electrodeposited Ni: microstructure and tensile properties," Acta Mater., vol. 50, no. 15, pp. 3957-3970, 2002.

[9] R. Schwaiger, B. Moser, M. Dao, N. Chollacoop, and S. Suresh, "Some critical experiments on the strain-rate sensitivity of nanocrystalline nickel," Acta Mater., vol. 51, no. 17, pp. 5159-5172, Oct. 2003.

[10] I. Chasiotis, C. Bateson, K. Timpano, A. S. McCarty, N. S. Barker, and J. R. Stanec, “Strain rate effects on the mechanical behavior of nanocrystalline Au films," Thin Solid Films, vol. 515, no. 6, pp. 3183-3189, Feb. 2007.

[11] Z. Jiang, X. Liu, G. Li, Q. Jiang, and J. Lian, "Strain rate sensitivity of a nanocrystalline Cu synthesized by electric brush plating," Appl. Phys. Lett., vol. 88, no. 14, p. 143115, 2006.

[12] H. S. Kim and Y. Estrin, "Phase mixture modeling of the strain rate dependent mechanical behavior of nanostructured materials," Acta Mater., vol. 53, no. 3, pp. 765-772, Feb. 2005.

[13] J. Rajagopalan and M. T. A. Saif, "Effect of microstructural heterogeneity on the mechanical behavior of nanocrystalline metal films," J. Mater. Res., vol. 26, no. 22, pp. 2826-2832, Nov. 2011.

[14] J. Rajagopalan, C. Rentenberger, H. Peter Karnthaler, G. Dehm, and M. T. A. Saif, "In situ TEM study of microplasticity and Bauschinger effect in nanocrystalline metals," Acta Mater., vol. 58, no. 14, pp. 4772-4782, Aug. 2010.

[15] E. Izadi and J. Rajagopalan, "Texture dependent strain rate sensitivity of ultrafine-grained aluminum films," Scr. Mater., vol. 114, pp. 65-69, Mar. 2016.

[16] D. Faurie, P.-O. Renault, E. Le Bourhis, and P. Goudeau, "Study of texture effect on elastic properties of Au thin films by X-ray diffraction and in situ tensile testing," Acta Mater., vol. 54, no. 17, pp. 4503-4513, Oct. 2006.

[17] H. Van Swygenhoven and S. Van Petegem, "In-situ mechanical testing during X-ray diffraction," Mater. Charact., vol. 78, pp. 47-59, Apr. 2013. 
[18] A. Kobler, A. Kashiwar, H. Hahn, and C. Kübel, "Combination of in situ straining and ACOM TEM: A novel method for analysis of plastic deformation of nanocrystalline metals," Ultramicroscopy, vol. 128, pp. 68-81, May 2013.

[19] M. Jin, A. M. Minor, E. A. Stach, and J. W. Morris Jr., "Direct observation of deformationinduced grain growth during the nanoindentation of ultrafine-grained $\mathrm{Al}$ at room temperature," Acta Mater., vol. 52, no. 18, pp. 5381-5387, Oct. 2004.

[20] D. Dingley, "Progressive steps in the development of electron backscatter diffraction and orientation imaging microscopy," J. Microsc., vol. 213, no. 3, pp. 214-224, Mar. 2004.

[21] T. E. Buchheit, J. D. Carroll, B. G. Clark, and B. L. Boyce, "Evaluating DeformationInduced Grain Orientation Change in a Polycrystal During In Situ Tensile Deformation using EBSD," Microsc. Microanal., vol. 21, no. 4, pp. 969-984, Aug. 2015.

[22] S. Zaefferer, "A critical review of orientation microscopy in SEM and TEM," Cryst. Res. Technol., vol. 46, no. 6, pp. 607-628, Jun. 2011.

[23] A. D. Darbal, K. J. Ganesh, X. Liu, S.-B. Lee, J. Ledonne, T. Sun, B. Yao, A. P. Warren, G. S. Rohrer, A. D. Rollett, P. J. Ferreira, K. R. Coffey, and K. Barmak, "Grain Boundary Character Distribution of Nanocrystalline Cu Thin Films Using Stereological Analysis of Transmission Electron Microscope Orientation Maps," Microsc. Microanal., vol. 19, no. 1, pp. 111-119, Feb. 2013.

[24] A. D. Darbal, M. Gemmi, J. Portillo, E. Rauch, and S. Nicolopoulos, "Nanoscale Automated Phase and Orientation Mapping in the TEM," Microsc. Today, vol. 20, no. 6, pp. 38-42, Nov. 2012.

[25] H. Idrissi, A. Kobler, B. Amin-Ahmadi, M. Coulombier, M. Galceran, J.-P. Raskin, S. Godet, C. Kübel, T. Pardoen, and D. Schryvers, "Plasticity mechanisms in ultrafine grained freestanding aluminum thin films revealed by in-situ transmission electron microscopy nanomechanical testing," Appl. Phys. Lett., vol. 104, no. 10, p. 101903, Mar. 2014.

[26] E. F. Rauch, J. Portillo, S. Nicolopoulos, D. Bultreys, S. Rouvimov, and P. Moeck, "Automated nanocrystal orientation and phase mapping in the transmission electron microscope on the basis of precession electron diffraction," Z. Krist., vol. 225, no. 2-3, pp. 103-109, 2010.

[27] A. C. Leff, C. R. Weinberger, and M. L. Taheri, "Estimation of dislocation density from precession electron diffraction data using the Nye tensor," Ultramicroscopy, vol. 153, pp. 9-21, Jun. 2015.

[28] A. C. Leff and M. L. Taheri, "Quantitative assessment of the driving force for twin formation utilizing Nye tensor dislocation density mapping," Scr. Mater., vol. 121, pp. 1417, Aug. 2016.

[29] F. Mompiou, M. Legros, A. Boé, M. Coulombier, J.-P. Raskin, and T. Pardoen, "Inter- and intragranular plasticity mechanisms in ultrafine-grained Al thin films: An in situ TEM study," Acta Mater., vol. 61, no. 1, pp. 205-216, Jan. 2013.

[30] J. H. Han, J. Rajagopalan, and M. T. A. Saif, "MEMS-Based Testing Stage to Study Electrical and Mechanical Properties of Nanocrystalline Metal Films," in SPIE, 2007, p. 64640C-64640C-8.

[31] R. Sarkar, C. Rentenberger, and J. Rajagopalan, "Electron Beam Induced Artifacts During in situ TEM Deformation of Nanostructured Metals," Sci. Rep., vol. 5, p. 16345, Nov. 2015.

[32] E. F. Rauch and M. Véron, "Automated crystal orientation and phase mapping in TEM," Mater. Charact., vol. 98, pp. 1-9, Dec. 2014. 
[33] D. S. Gianola, S. Van Petegem, M. Legros, S. Brandstetter, H. Van Swygenhoven, and K. J. Hemker, "Stress-assisted discontinuous grain growth and its effect on the deformation behavior of nanocrystalline aluminum thin films," Acta Mater., vol. 54, no. 8, pp. 22532263, May 2006.

[34] F. Mompiou, D. Caillard, M. Legros, and H. Mughrabi, "In situ TEM observations of reverse dislocation motion upon unloading in tensile-deformed UFG aluminium," Acta Mater., vol. 60, no. 8, pp. 3402-3414, May 2012.

[35] F. Mompiou and M. Legros, "Quantitative grain growth and rotation probed by in-situ TEM straining and orientation mapping in small grained Al thin films," Scr. Mater., vol. 99, pp. 5-8, Apr. 2015.

[36] T. J. Rupert, D. S. Gianola, Y. Gan, and K. J. Hemker, "Experimental Observations of Stress-Driven Grain Boundary Migration,” Science, vol. 326, no. 5960, pp. 1686-1690, Dec. 2009.

[37] A. Rajabzadeh, F. Mompiou, M. Legros, and N. Combe, "Elementary Mechanisms of Shear-Coupled Grain Boundary Migration," Phys. Rev. Lett., vol. 110, no. 26, p. 265507, Jun. 2013.

[38] N. Ledermann, P. Muralt, J. Baborowski, S. Gentil, K. Mukati, M. Cantoni, A. Seifert, and N. Setter, " $\left\{\begin{array}{lll}1 & 0 & 0\end{array}\right\}$-Textured, piezoelectric $\mathrm{Pb}(\mathrm{Zrx}, \mathrm{Ti1}-\mathrm{x}) \mathrm{O} 3$ thin films for MEMS: integration, deposition and properties," Sens. Actuators Phys., vol. 105, no. 2, pp. 162-170, Jul. 2003.

[39] R. G. Polcawich, J. S. Pulskamp, D. Judy, P. Ranade, S. Trolier-McKinstry, and M. Dubey, "Surface Micromachined Microelectromechancial Ohmic Series Switch Using Thin-Film Piezoelectric Actuators," IEEE Trans. Microw. Theory Tech., vol. 55, no. 12, pp. 26422654, Dec. 2007.

[40] D. C. Bufford, D. Stauffer, W. M. Mook, S. A. Syed Asif, B. L. Boyce, and K. Hattar, "High Cycle Fatigue in the Transmission Electron Microscope," Nano Lett., vol. 16, no. 8, pp. 4946-4953, Aug. 2016. 


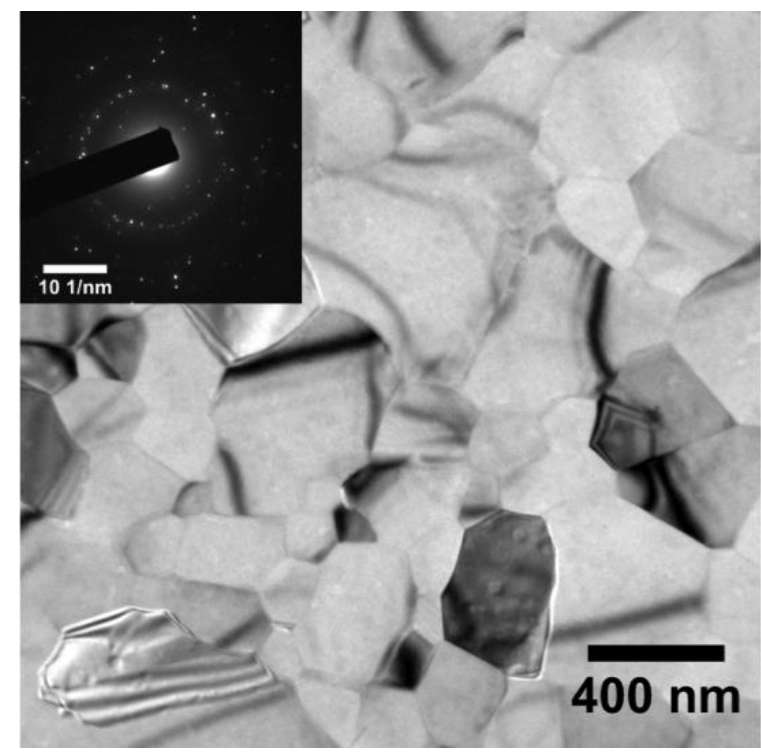

Figure 1: Bright-field TEM image of a $200 \mathrm{~nm}$ thick, non-textured aluminum film with a mean grain size of $180 \mathrm{~nm}$. Selected area diffraction of the film showing the lack of texture (inset).

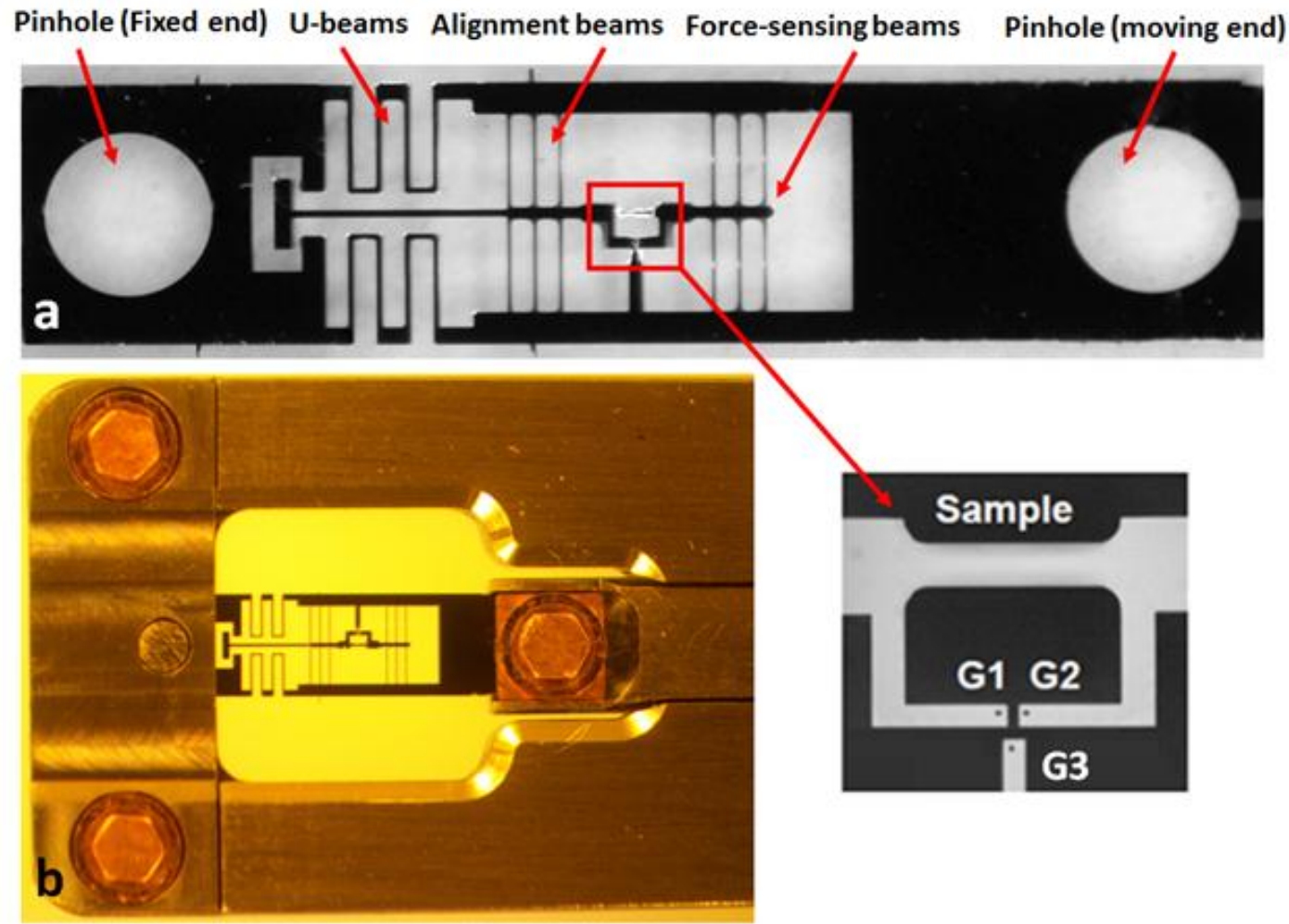

Figure 2: a) MEMS device for performing ex situ and in situ experiments on freestanding metal film samples. The stress and nominal strain on the sample is obtained by tracking the displacement of gauges G1, G2 and G3. b) Optical micrograph of the MEMS device mounted on the Gatan TEM straining holder. 


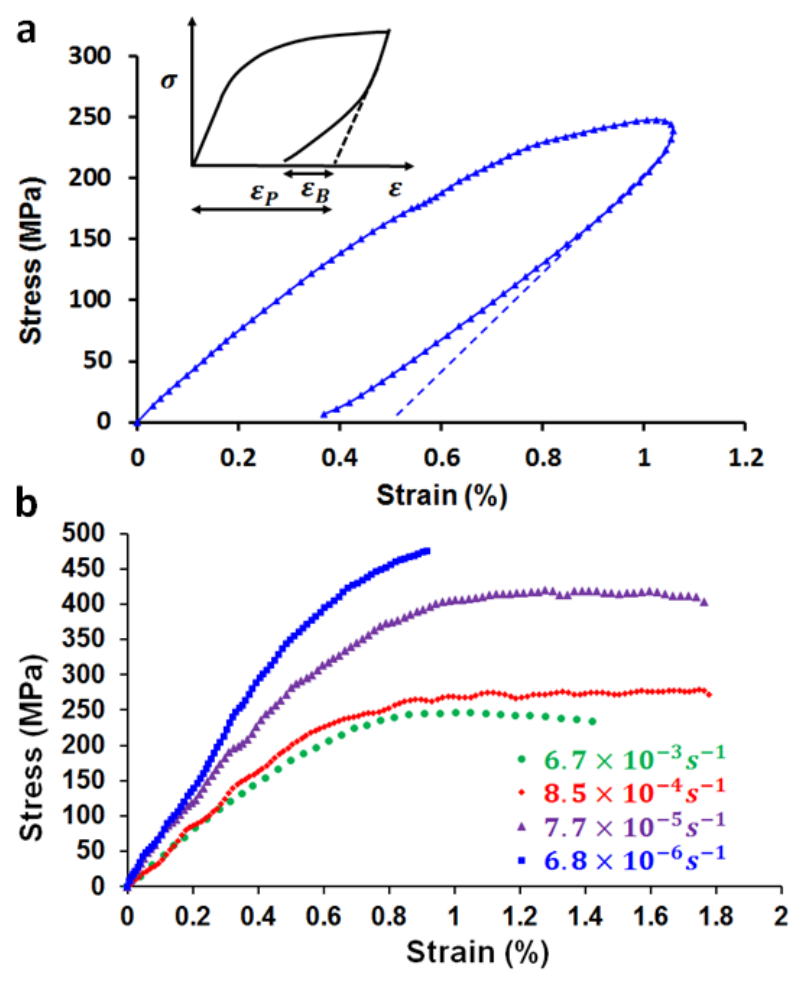

Figure 3: a) Stress-strain response of the $200 \mathrm{~nm}$ thick non-textured Al film during quasi-static loading and unloading. The dashed blue line represents the linear elastic unloading path. b) Stress-strain response of a similar non-textured film (thickness $240 \mathrm{~nm}$, mean grain size $285 \mathrm{~nm}$ ) at different strain rates (adapted from [15]).

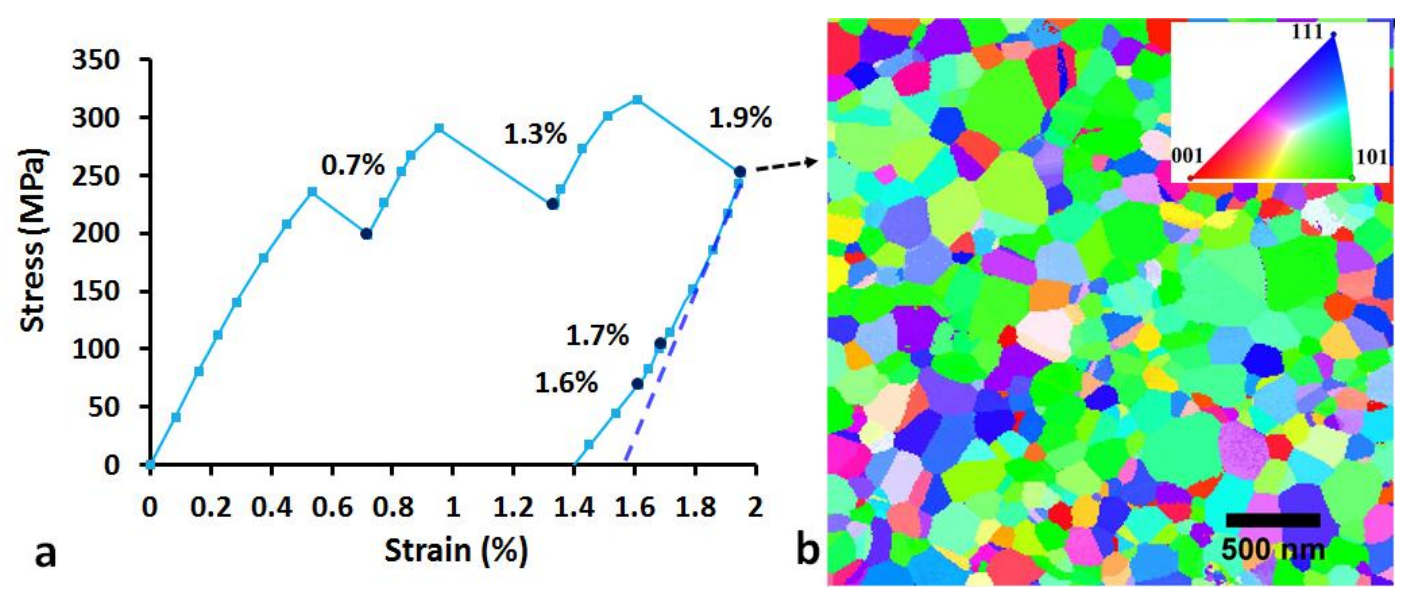

Figure 4: a) Stress-strain response of the $200 \mathrm{~nm}$ non-textured Al film during in situ ACOMTEM experiment. Consecutive data points are joined by straight lines in the plot. The orientation maps were acquired at three points during loading $(0.7 \%, 1.3 \%$ and $1.9 \%$ strain) and two points during unloading (1.7\% and 1.6\% strain). b) An ACOM- map showing the color-coded out-ofplane orientation of the grains in the scanning area. 


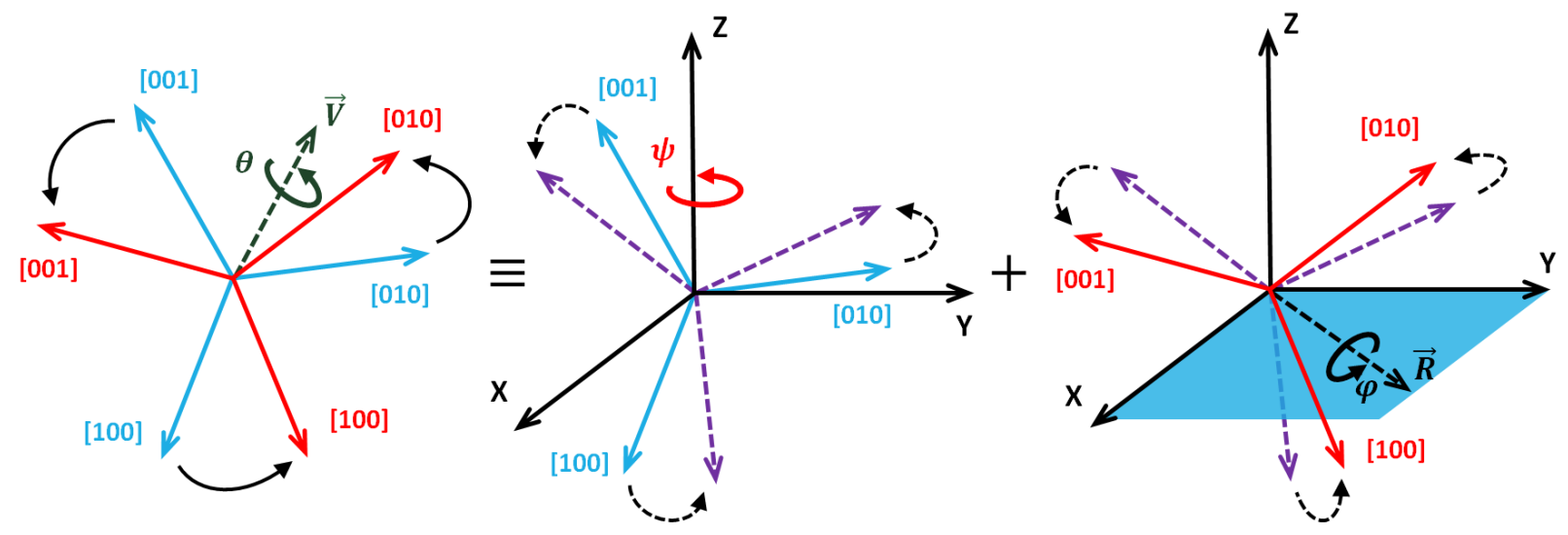

Figure 5: Schematic of the analysis of grain rotations from the ACOM-TEM data. The red axes represent the reference crystallographic axes of a grain. The blue axes represent the crystallographic axes of the grain in the deformed state where it has undergone rotation. A rotation $\theta$ about the direction $\vec{V}$ transforms the axes in the deformed configuration to the reference configuration. This axes transformation can also be accomplished by two rotations $-\mathrm{a}$ in-plane rotation $\psi$ about $\mathrm{Z}$-axis (normal to the film) followed by an out-of-plane rotation about an arbitrary direction $\vec{R}$, which lies in the X-Y plane. 

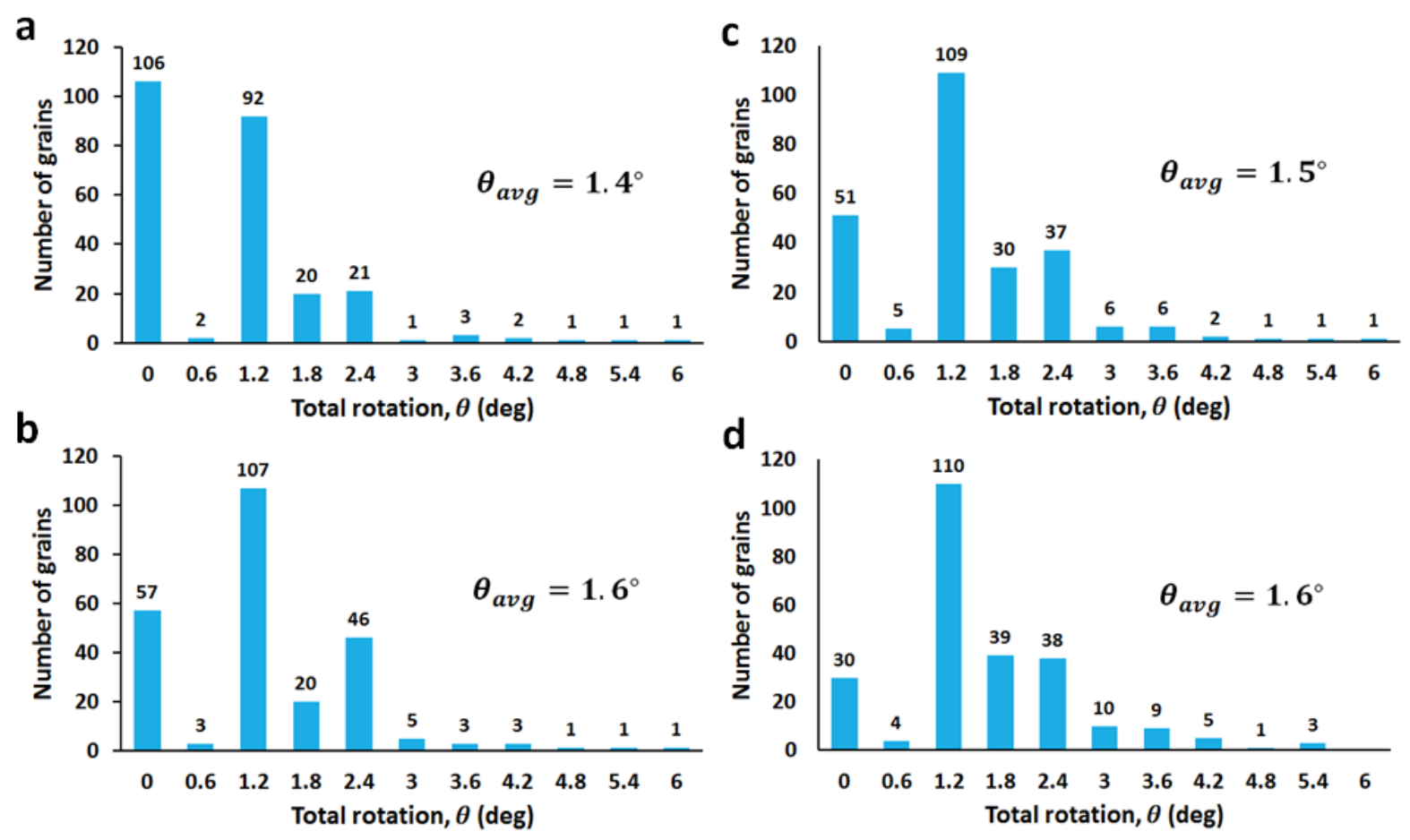

Figure 6: Histogram of the total rotation $(\theta)$ induced in approximately 250 grains during loading from a) $0.7 \%$ strain to $1.3 \%$ strain, and b) $0.7 \%$ strain to $1.9 \%$ strain. Histogram of grain rotations at the two unloading points, c) $1.7 \%$ strain and d) $1.6 \%$ strain. Note that the grain rotations for the unloading points are also calculated with respect to reference configuration at $0.7 \%$ strain during loading. A few grains that exhibited rotations greater than $6^{\circ}$ are not included in the histograms.
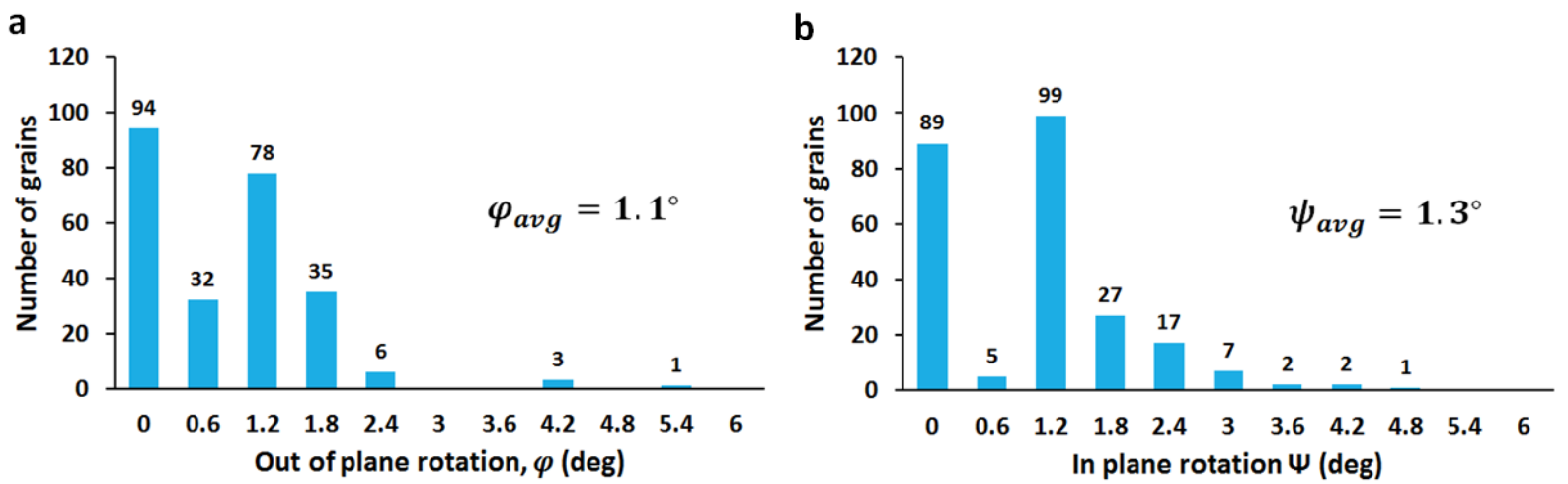

Figure 7: a) Histogram of out-of-plane grain rotations during loading from $0.7 \%$ strain to $1.9 \%$ strain. b) Histogram of in-plane grain rotations during loading from $0.7 \%$ strain to $1.9 \%$ strain. 


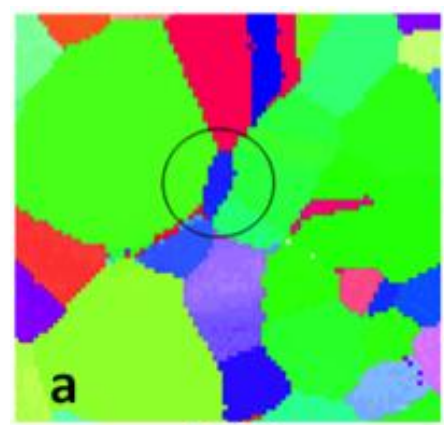

$1.3 \% \mathrm{~L}$

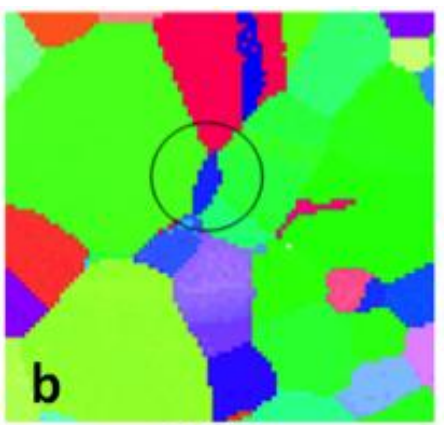

$1.9 \% \mathrm{~L}$

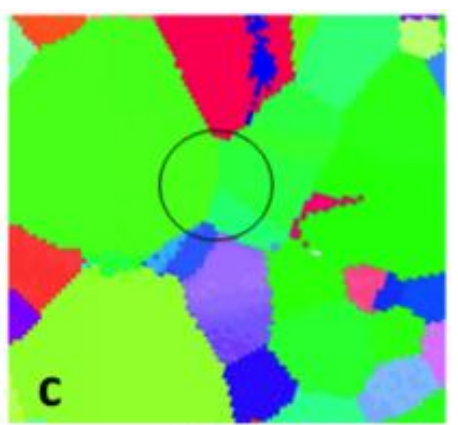

$1.7 \%$ UL

Figure 8: Progressive reduction in size and complete annihilation of a grain (marked by the black circle) during loading and unloading. ' $\mathrm{L}$ ' denotes loading and ' $\mathrm{UL}$ ' denotes unloading in the figure.

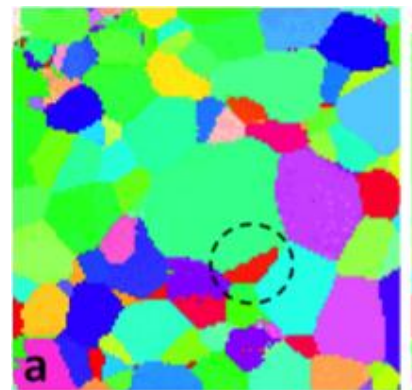

$0.7 \% \mathrm{~L}$

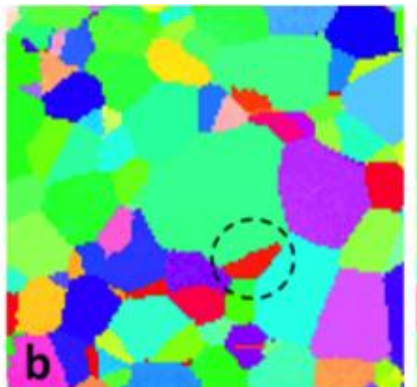

$1.3 \% \mathrm{~L}$

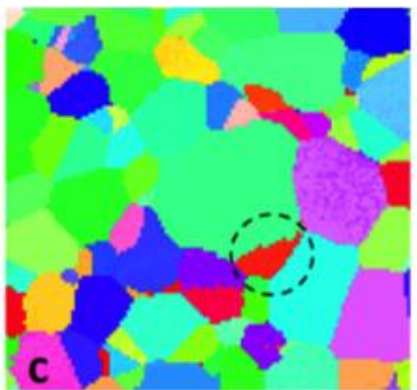

$1.9 \% \mathrm{~L}$

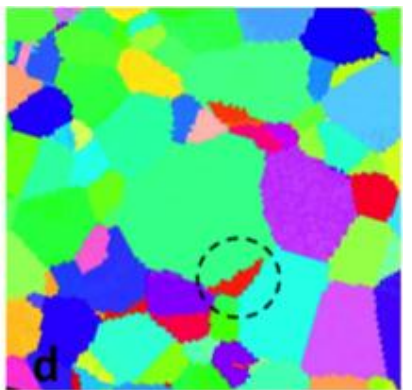

$1.7 \%$ UL

Figure 9: Reversible change in size of a grain (marked by the black dashed circle) during loading and unloading. The size of the grain increased during loading but reduced as the sample was unloaded. ' $L$ ' denotes loading and 'UL' denotes unloading in the figure.

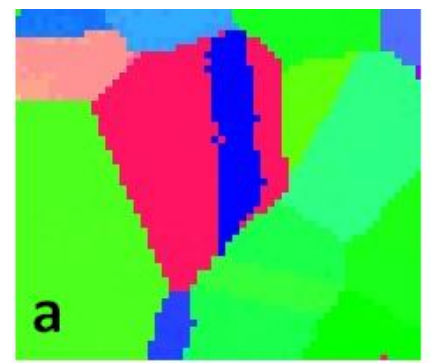

$1.3 \% \mathrm{~L}$

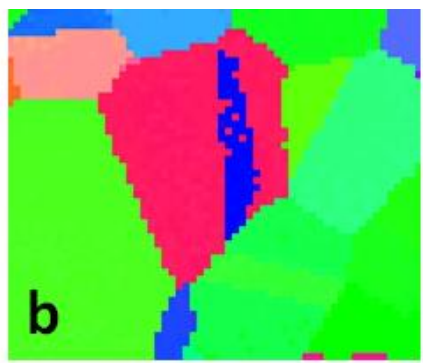

$1.9 \% \mathrm{~L}$

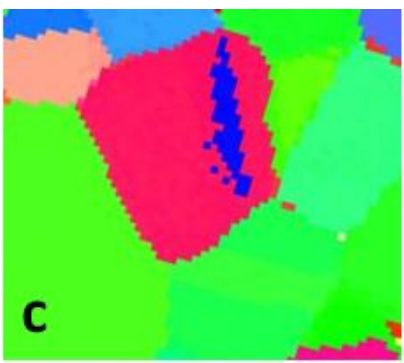

$1.6 \%$ UL

Figure 10: Detwinning in a grain during loading and unloading. ' $L$ ' denotes loading and 'UL' denotes unloading in the figure. 


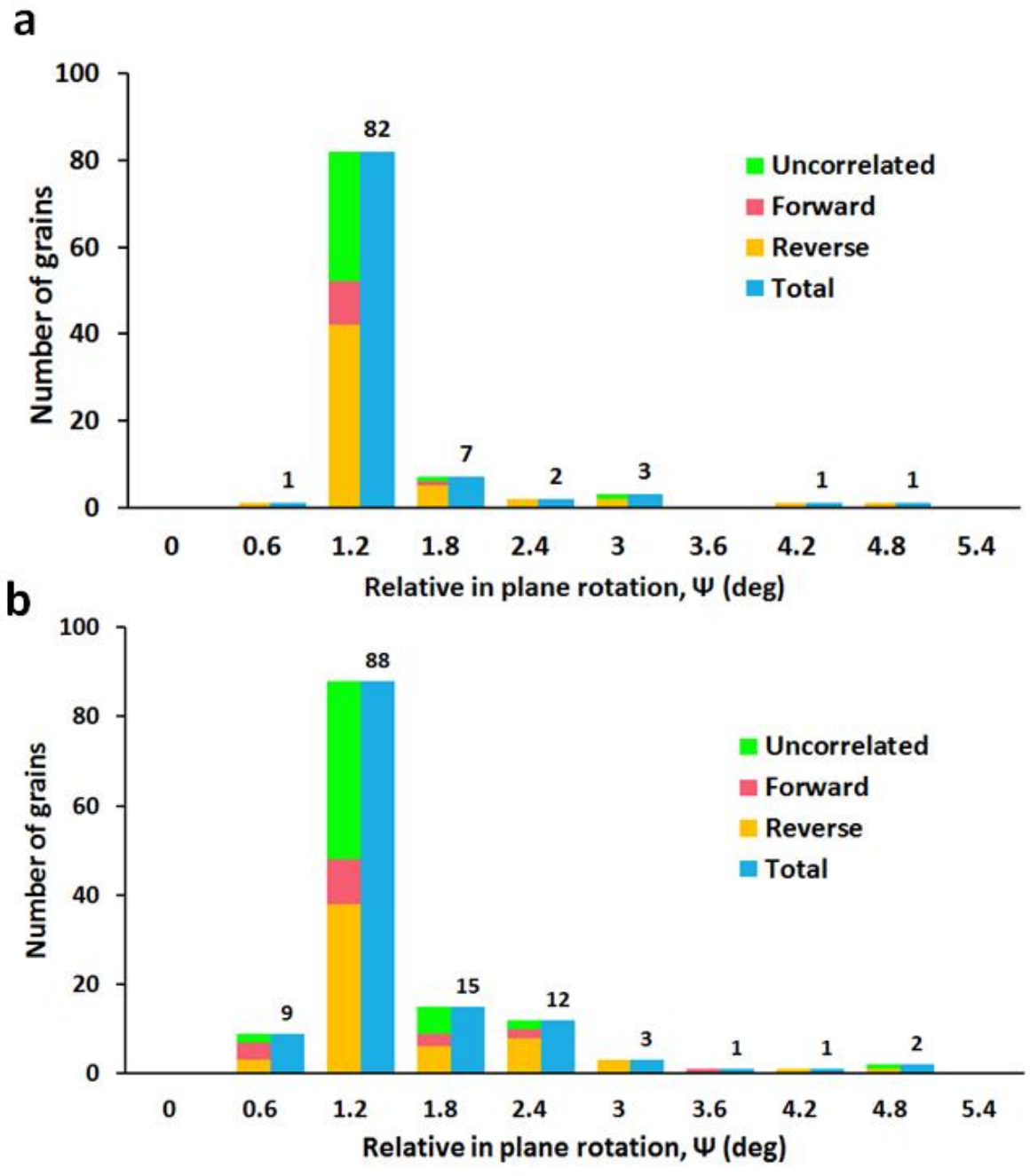

Figure 11: Histogram of grains that exhibited in-plane rotations during unloading with respect to the end of loading. The classifications are based on the scheme explained in the text. Figure (a) corresponds to $1.7 \%$ strain during unloading whereas (b) corresponds to $1.6 \%$ strain during unloading. Grains that showed zero relative rotation are not included in the histograms. 
